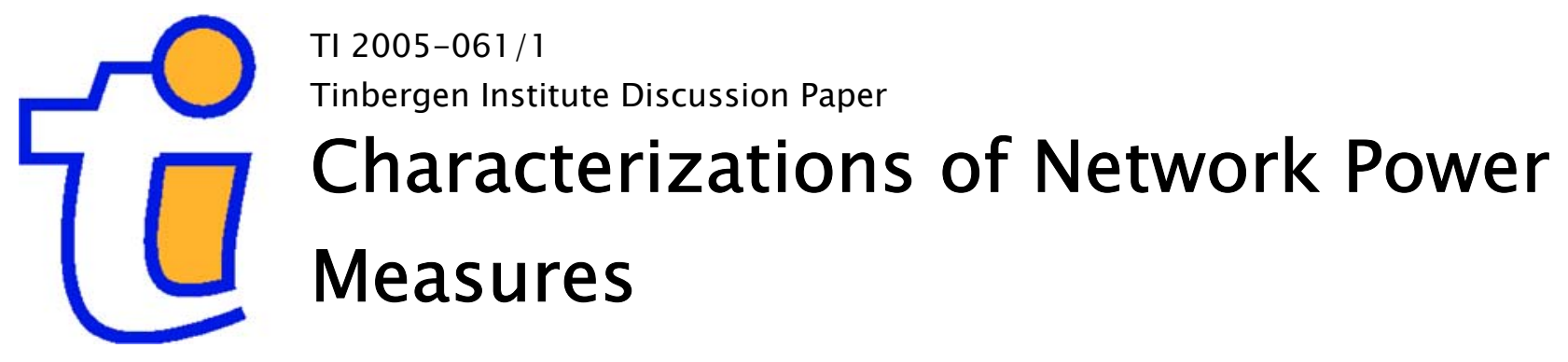

René van den Brink ${ }^{1}$

Peter Borm²

Ruud HendrickX²

Guillermo Owen ${ }^{3}$

1 Department of Econometrics, Free University Amsterdam, and Tinbergen Institute,

2 CentER, and Department of Econometrics and Operations Research, Tilburg University,

${ }_{3}^{3}$ Department of Mathematics, Naval Postgraduate School, Monterey, California, USA. 


\section{Tinbergen Institute}

The Tinbergen Institute is the institute for economic research of the Erasmus Universiteit Rotterdam, Universiteit van Amsterdam, and Vrije Universiteit Amsterdam.

Tinbergen Institute Amsterdam

Roetersstraat 31

1018 WB Amsterdam

The Netherlands

Tel.: $\quad+31(0) 205513500$

Fax: $\quad+31(0) 205513555$

Tinbergen Institute Rotterdam

Burg. Oudlaan 50

3062 PA Rotterdam

The Netherlands

Tel.: $\quad+31(0) 104088900$

Fax: $\quad+31(0) 104089031$

Please send questions and/or remarks of nonscientific nature to driessen@tinbergen.nl.

Most TI discussion papers can be downloaded at http://www.tinbergen.nl. 


\title{
Characterizations of Network Power Measures
}

\author{
René van den Brink*, Peter Borm $;$ Ruud Hendrickx; Guillermo Owen ${ }^{\S}$ \\ June 8, 2005
}

\begin{abstract}
A symmetric network consists of a set of positions and a set of bilateral links between these positions. Examples of such networks are exchange networks, communication networks, disease transmission networks, control networks etc. For every symmetric network we define a cooperative transferable utility game that measures the "power" of each coalition of positions in the network. Applying the Shapley value to this game yields a network power measure, the $\beta$-measure, which reflects the power of the individual positions in the network.

Applying this power distribution method iteratively yields a limit distribution, which turns out to equal the well-known degree measure. We compare the $\beta$ measure and degree measure by providing characterizations, which differ only in the normalization that is used.
\end{abstract}

Keywords: symmetric networks, network power, stationary power distribution, $\beta$-measure, degree measure.

JEL code: C71, Z13.

*Corresponding author: Department of Econometrics and Tinbergen Institute, Free University, De Boelelaan 1105, 1081 HV Amsterdam, The Netherlands. E-mail: jrbrink@feweb.vu.nl

${ }^{\ddagger}$ CentER and Department of Econometrics and Operations Research, Tilburg University, The Netherlands.

$\S^{\S}$ Department of Mathematics, Naval Postgraduate School, Monterey, California, USA. 


\section{Introduction}

An undirected graph, which consists of a set of positions and a set of links between pairs of positions, can represent various types of networks. Examples of such symmetric networks, in which the roles of the two positions on each link are symmetric, are exchange networks, communication networks, disease transmission networks and control networks. On the other hand, in asymmetric networks the positions on a link have different roles. One can think of buyer-seller networks or firm structures. The purpose of this paper is to measure "power" or "control" of positions in symmetric networks.

For every symmetric network we define a cooperative transferable utility game that measures the power of coalitions of positions. Applying the Shapley value (Shapley (1953)) to this network power game yields the $\beta$-measure, which is discussed in van den Brink and Gilles (2000) and van den Brink and Borm (2002) for asymmetric networks. The idea behind the $\beta$-measure is that each position in a network has an initial weight equal to 1 , and measuring power is seen as fairly redistributing this weight to all its neighbours. This measure fits well with power dependence theory as developed by Emerson (1962) since the power value of a position decreases when its neighbours have more other neighbours.

Instead of taking initial weights equal to 1 , it seems natural to take weights that already reflect some power of the positions. In this way one obtains weighted $\beta$-measures. Similar as done in Borm, van den Brink and Slikker (2002) for asymmetric networks, we consider a sequence of weighted $\beta$-measures. Starting with the (unweighted) $\beta$-measure, we compute in each step a new weighted $\beta$-measure, taking the outcome of the previous step as input weights. We show that this sequence has a limit, which equals the wellknown degree measure for symmetric networks. This degree measure assigns to every position just its number of direct neighbours.

At first sight the degree measure only seems to take the direct relations of a position into account in determining its power value, whereas the $\beta$-measure takes account of (some) indirect relations. Therefore, the degree measure is usually considered to be a local power measure. However, since the degree measure is the limit of the sequence of weighted $\beta$-measures, it can be seen as a global measure within the context of power dependence theory.

Besides characterizing the degree measure as the limit of the weighted $\beta$-measures, we provide axiomatic characterizations of the $\beta$-measure and degree measure. These characterizations, which form the main results of this paper, are based on local graph- 
manipulating properties and differ only in the normalization that is used.

The paper is organized as follows. In Section 2 we briefly discuss some graph and game theoretic preliminaries. In Section 3 we introduce network power games and introduce the $\beta$-measure for symmetric networks. We also discuss the sequence of weighted $\beta$ measures and show that its limit equals the degree measure. The characterizations of the $\beta$-measure and degree measure are provided in Section 4.

\section{Preliminaries}

In this section we discuss some graph and game theoretic preliminaries. A symmetric network or undirected graph is a pair $(N, G)$ where $N$ is a finite set of positions or nodes, and $G \subseteq\{\{i, j\} \mid i, j \in N, i \neq j\}$ is a set of symmetric edges or links between these positions. So, we assume the networks to be irreflexive, i.e., $(i, i) \notin G$ for all $i \in N$. The collection of all (irreflexive) networks is denoted by $\mathcal{G}$. We often refer to these just as graphs.

For every graph $(N, G) \in \mathcal{G}$ and set of positions $T \subseteq N$, the induced subgraph $(T, G(T))$ is given by $G(T)=\{\{i, j\} \in G \mid\{i, j\} \subseteq T\}$. A network $(N, G)$ is connected if for every pair of positions $i, j \in N$ there exists a sequence of positions $h_{1}, \ldots, h_{p}$ such that $h_{1}=i, h_{p}=j$, and $\left\{h_{k}, h_{k+1}\right\} \in G$ for all $k \in\{1, \ldots, p-1\}$. A set of positions $T \subseteq N$ is a component in $(N, G) \in \mathcal{G}$ if it is a maximally connected subset of $N$ in $(N, G)$, i.e., if the graph $(T, G(T))$ is connected and for every $i \in N \backslash T$ the graph $(T \cup\{i\}, G(T \cup\{i\}))$ is not connected. If $\{i, j\} \in G$, then positions $i$ and $j$ are called neighbours and are incident with the edge $\{i, j\}$. By $R_{(N, G)}(i)$ we denote the set of all neighbours of position $i \in N$ in network $(N, G)$ by

$$
R_{(N, G)}(i)=\{j \in N \mid\{i, j\} \in G\} .
$$

For a set of positions $S \subseteq N$ we denote $R_{(N, G)}(S)=\bigcup_{i \in S} R_{(N, G)}(i)$. If $R_{(N, G)}(i)=\emptyset$, then position $i$ is called an isolated position. Position $i \in N$ is called a pending position if $\left|R_{(N, G)}(i)\right|=1$. We denote the set of isolated positions in network $(N, G)$ by $I(N, G)$ and the set of pending positions by $P(N, G)$.

Finally, a network power measure for symmetric networks is a mapping $p$ that assigns to every network $(N, G) \in \mathcal{G}$ an $|N|$-dimensional vector $p(N, G) \in \mathbb{R}^{N}$. We refer to this vector as a network power distribution for $(N, G)$. A well-known network power measure is the degree measure, which assigns to every position in a network the number 
of its neighbours. The degree measure thus is the power measure $d$ given by

$$
d_{i}(N, G)=\left|R_{(N, G)}(i)\right| \text { for all } i \in N
$$

A (finite) cooperative game with transferable utility (or simply TU-game) is a pair $(N, v)$ with finite set $N$ of players and characteristic function $v: 2^{N} \rightarrow \mathbb{R}$ satisfying $v(\emptyset)=0$. A (single valued) solution for TU-games is a function $f$ that assigns to every TUgame $(N, v)$ an $|N|$-dimensional vector $f(N, v) \in \mathbb{R}^{N}$, representing a distribution of payoffs to the players. A well-known solution is the Shapley value (Shapley (1953)), which equally distributes the dividends $\Delta_{v}(S)$ (see Harsanyi (1959)) over all players in coalition $S \subseteq N, S \neq \emptyset$ :

$$
S h_{i}(N, v)=\sum_{\{S \subseteq N \mid i \in S\}} \frac{\Delta_{v}(S)}{|S|} \text { for all } i \in N
$$

where $\Delta_{v}(S)=v(S)$ if $|S|=1$, and recursively $\Delta_{v}(S)=v(S)-\sum_{\{T \subsetneq S \mid T \neq \emptyset\}} \Delta_{v}(T)$ for all $S \subseteq N,|S| \geq 2$. For every $T \subseteq N, T \neq \emptyset$, the unanimity game $u_{T}$ is given by $u_{T}(S)=1$ if $T \subseteq S$, and $u_{T}(S)=0$ otherwise. Every characteristic function $v: 2^{N} \rightarrow \mathbb{R}$ can be written as a linear combination of unanimity games in a unique way by $v=\sum_{\{T \subseteq N \mid T \neq \emptyset\}} \Delta_{v}(T) u_{T}$.

\section{Network power games and measures}

In order to measure power or control in networks, we assign to every network $(N, G) \in \mathcal{G}$ a cooperative game with transferable utility $(N, v)$, whose set of players $N$ corresponds to the set of positions in the network. In cooperative game theoretic tradition we take a conservative approach to measuring power of coalitions by assigning to every coalition of positions $S \subseteq N$ the number of neighbours of $S$ that have no neighbours outside $S$. The network power game $\left(N, v_{G}\right)$ corresponding to $(N, G) \in \mathcal{G}$ thus is given by

$$
v_{G}(S)=\left|\left\{j \in R_{(N, G)}(S) \mid R_{(N, G)}(j) \subseteq S\right\}\right| \text { for all } S \subseteq N .
$$

Note that $v_{G}(N)=|N \backslash I(N, G)|$ for all $(N, G) \in \mathcal{G}$. The dividends of $v_{G}$ are given by

$$
\Delta_{v_{G}}(S)=\left|\left\{j \in N \mid R_{(N, G)}(j)=S\right\}\right| \text { for all } S \subseteq N, S \neq \emptyset .
$$

Hence, this game can be decomposed as $v_{G}=\sum_{i \in R_{(N, G)}(N)} u_{R_{(N, G)}(i)}$. So, every network power game is totally positive meaning that it can be expressed as a nonnegative sum of unanimity games. As a corollary, a network power game is convex meaning that $v(S)+v(T) \leq v(S \cup T)+v(S \cap T)$ for all $S, T \subseteq N$. 
The Shapley value of a network power game can be seen as a network power distribution of the underlying network. The corresponding power measure is called the $\beta$-measure:

$$
\beta(N, G)=S h\left(N, v_{G}\right) \text { for all }(N, G) \in \mathcal{G}
$$

Proposition 3.1 For every $(N, G) \in \mathcal{G}$ we have

$$
\beta_{i}(N, G)=\sum_{j \in R_{(N, G)}(i)} \frac{1}{\left|R_{(N, G)}(j)\right|} \text { for all } i \in N \text {. }
$$

Proof: Using (2), we obtain

$$
\begin{aligned}
\beta_{i}(N, G) & =S h_{i}\left(N, v_{G}\right)=\sum_{\{S \subseteq N \mid i \in S\}} \frac{\Delta_{v_{G}}(S)}{|S|}=\sum_{\{S \subseteq N \mid i \in S\}} \frac{\left|\left\{j \in N \mid R_{(N, G)}(j)=S\right\}\right|}{|S|} \\
& =\sum_{\{S \subseteq N \mid i \in S\}} \sum_{\left\{j \in N \mid R_{(N, G)}(j)=S\right\}} \frac{1}{\left|R_{(N, G)}(j)\right|}=\sum_{j \in R_{(N, G)}(i)} \frac{1}{\left|R_{(N, G)}(j)\right|} .
\end{aligned}
$$

The idea behind the $\beta$-measure is that every position in a network has an initial weight equal to 1 , and each of its neighbours receives an equal share of this weight. Instead of taking initial weights equal to 1 , it seems natural to take weights that already reflect the power of the positions. If we take the $\beta$-measure as initial weights, we obtain the second order measure $\beta^{2}$. Of course, this second order measure can be used as new input weights, and so on, yielding higher order measures. Starting with

$$
\beta_{i}^{0}(N, G)=1 \text { for all } i \in N
$$

we recursively define the measures

$$
\beta_{i}^{t}(N, G)=\sum_{j \in R_{(N, G)}(i)} \frac{\beta_{j}^{t-1}(N, G)}{\left|R_{(N, G)}(j)\right|} \text { for all } i \in N, t \in\{1,2, \ldots\} .
$$

In particular, $\beta^{1}(N, G)=\beta(N, G)$. This sequence of measures has a limit, which is a stationary power distribution. A power distribution $p \in \mathbb{R}^{N}$ is a stationary power distribution of (3) if redistributing these weights according to (3) yields the same weights:

$$
p_{i}(N, G)=\sum_{j \in R_{(N, G)}(i)} \frac{p_{j}(N, G)}{\left|R_{(N, G)}(j)\right|} \text { for all } i \in N .
$$

Borm, van den Brink and Slikker (2002) define a sequence similar to (3) for "directed" or "asymmetric" networks $(N, D)$ with $D \subseteq N \times N$, and show that it has a limit, which is 
also a stationary distribution. Defining for every undirected network $(N, G) \in \mathcal{G}$ the corresponding directed network $(N, D(G))$ with $D(G)=\{(i, j) \in N \times N \mid\{i, j\} \in G\}$, the existence of a stationary distribution of (3) can be shown in a similar way as their result. Moreover, since for every $(N, G) \in \mathcal{G}$ and $i \in N$ we have that $\sum_{j \in R_{(N, G)}(i)} \frac{d_{j}(N, G)}{\left|R_{(N, G)}(j)\right|}=$ $\sum_{j \in R_{(N, G)}(i)} \frac{\left|R_{(N, G)}(j)\right|}{\left|R_{(N, G)}(j)\right|}=\left|R_{(N, G)}(i)\right|=d_{i}(N, G)$, the degree measure yields a stationary power distribution of $(3)$. In case the network $(N, G)$ is connected, the corresponding directed network $(N, D(G))$ is strongly connected ${ }^{1}$, and it follows from standard results on such networks (see, e.g., Berger (1993)) that (3) has a unique stationary power distribution ${ }^{2}$.

Proposition 3.2 For every $(N, G) \in \mathcal{G}$ the sequence defined by (3) has a limit, which is equal to the degree measure of $(N, G)$. This limit is a stationary power distribution of $(N, G)$. Moreover, if the network $(N, G)$ is connected, then this is the unique stationary power distribution.

\section{Characterizations}

In this section we provide characterizations of the $\beta$-measure and the degree measure. The first property is a normalization determining the total value of "power" to be distributed. Since we want to measure how well positions in a network are connected with other positions, we normalize power such that the total weight that is distributed over the positions in a network is equal to the number of non-isolated positions. Since this boils down to efficiency of a solution for the corresponding network power game, we refer to this property as efficiency.

Efficiency: For every $(N, G) \in \mathcal{G}$ it holds that $\sum_{i \in N} p_{i}(N, G)=|N \backslash I(N, G)|$.

The second property is anonymity, which says that two similar positions in a network have the same power value. For a network $(N, G) \in \mathcal{G}$ and permutation $\pi: N \rightarrow N$, we define the permuted network $(N, \pi G) \in \mathcal{G}$ by $\{\pi(i), \pi(j)\} \in \pi G$ if and only if $\{i, j\} \in G$.

Anonymity: For every $(N, G) \in \mathcal{G}$ and permutation $\pi: N \rightarrow N$ it holds that $p_{i}(N, G)=p_{\pi(i)}(N, \pi G)$ for all $i \in N$.

\footnotetext{
${ }^{1} \mathrm{~A}$ directed graph $(N, D)$ is strongly connected if for each pair of positions $i, j \in N, i \neq j$ there is a sequence of nodes $i_{1}, \ldots, i_{p}$ such that $i_{1}=i, i_{p}=j$ and $\left(i_{k}, i_{k+1}\right) \in D$ for all $k \in\{1, \ldots, p-1\}$.

${ }^{2}$ If the network is not connected then it can be shown that in every stationary power distribution the power in every component is distributed proportional to the degrees of the nodes in that component.
} 
The third property is a boundary condition, which states that the power value of a position never exceeds the number of its neighbours, and is at least equal to the number of its neighbours that have no other neighbours.

Reasonability: For every $(N, G) \in \mathcal{G}$ and $i \in N$ it holds that $\mid\left\{j \in R_{(N, G)}(i) \mid R_{(N, G)}(j)=\right.$ $\{i\}\}\left|\leq p_{i}(N, G) \leq\right| R_{(N, G)}(i) \mid$.

The next three properties express that power is determined locally in the network. The first of these states that the power value of a position does not change if we delete or add relations between positions that do not contain either this position itself or any of its direct neighbours.

Non-neighbourhood independence: Let $(N, G),\left(N, G^{\prime}\right) \in \mathcal{G}$ and $i \in N$ be such that $R_{(N, G)}(j)=R_{\left(N, G^{\prime}\right)}(j)$ for all $j \in\{i\} \cup R_{(N, G)}(i)$. Then $p_{i}(N, G)=p_{i}\left(N, G^{\prime}\right)$.

The fifth property states that cutting an edge into two pieces and putting two new positions at the two endings does not change the power of the positions that are not incident with the edge that is cut.

Edge cutting independence: Let $(N, G)$ and $\left(N^{\prime}, G^{\prime}\right)$ be such that $N^{\prime}=N \cup\{r, s\}$ with $r, s \notin N$, and $G^{\prime}=(G \backslash\{h, j\}) \cup\{\{h, r\},\{j, s\}\}$ for $\{h, j\} \in G$. Then $p_{i}(N, G)=$ $p_{i}\left(N^{\prime}, G^{\prime}\right)$ for all $i \in N \backslash\{h, j\}$.

Finally, the sixth property states that adding a new position to an existing position changes the power value of each neighbour of the existing position by the same amount.

Pending node addition: Let $(N, G)$ and $\left(N^{\prime}, G^{\prime}\right)$ be such that $N^{\prime}=N \cup\{g\}$ with $g \notin N$, and $G^{\prime}=G \cup\{h, g\}$ for some $h \in N$. Then $p_{i}(N, G)-p_{i}\left(N^{\prime}, G^{\prime}\right)=$ $p_{j}(N, G)-p_{j}\left(N^{\prime}, G^{\prime}\right)$ for all $i, j \in R_{(N, G)}(h)$.

It is readily verified that the $\beta$-measure satisfies the six properties introduced above. To prove uniqueness, we start by showing that the first three properties uniquely determine the $\beta$-measure for star networks. We call a network $(N, G) \in \mathcal{G}$ a star network if there exists an $h \in N$ with $h \in\{i, j\}$ for all $\{i, j\} \in G$. We call $h$ a central position in this star. (Note that in our context a star is not necessarily connected, but there is at most one component that does not consist of one isolated position.)

Lemma 4.1 If a power measure $p$ on $\mathcal{G}$ satisfies efficiency, anonymity and reasonability, and $(N, G) \in \mathcal{G}$ is a star, then $p(N, G)=\beta(N, G)$. 
Proof: Let $(N, G) \in \mathcal{G}$ be a star with central position $h$. Reasonability implies that $p_{i}(N, G)=0$ for all $i \in I(N, G)=N \backslash\left(\{h\} \cup R_{(N, G)}(h)\right)$, and $p_{h}(N, G)=\left|R_{(N, G)}(h)\right|$. It then follows from efficiency that $\sum_{j \in R_{(N, G)}(h)} p_{j}(N, G)=\left|R_{(N, G)}(h)\right|+1-\left|R_{(N, G)}(h)\right|=1$. Anonymity then yields $p_{j}(N, G)=\frac{1}{\left|R_{(N, G)}(h)\right|}$ for all $j \in R_{(N, G)}(h)$. Hence, $p(N, G)=$ $\beta(N, G)$.

Adding non-neighbourhood independence to these properties uniquely determines the values for isolated and pending positions in any network.

Lemma 4.2 If a power measure $p$ on $\mathcal{G}$ satisfies efficiency, anonymity, reasonability and non-neighbourhood independence, then $p_{i}(N, G)=\beta_{i}(N, G)$ for all $(N, G) \in \mathcal{G}$ and all $i \in I(N, G) \cup P(N, G)$.

Proof: Let $(N, G) \in \mathcal{G}$ and let $i \in N$. Define $G_{i}$ to be the induced subgraph on all positions at distance at most 2 to $i$ :

$$
G_{i}=\left\{\{h, j\} \in G \mid h \in R_{(N, G)}(i)\right\}
$$

If $i \in P(N, G) \cup I(N, G)$, then $\left(N, G_{i}\right)$ is a star network and $p_{i}\left(N, G_{i}\right)=\beta_{i}\left(N, G_{i}\right)$ by Lemma 4.1. It then follows from non-neighbourhood independence that $p_{i}(N, G)=$ $p_{i}\left(N, G_{i}\right)=\beta_{i}\left(N, G_{i}\right)=\beta_{i}(N, G)$.

Next we show uniqueness for networks that can be seen as "stars" with two central positions. We call a network $(N, G) \in \mathcal{G}$ a double-centered star if there exists an edge $\{h, i\} \in G$ such that $\{h, i\} \cap\{g, j\} \neq \emptyset$ for all $\{g, j\} \in G$ and $R_{(N, G)}(h) \cap R_{(N, G)}(i)=\emptyset$. We call $h$ and $i$ the central positions in this double-centered star. Note that a star is a double-centered star in which (at least) one of the central positions is pending.

Lemma 4.3 If a power measure $p$ on $\mathcal{G}$ satisfies efficiency, anonymity, reasonability, non-neighbourhood independence and pending node addition, and $(N, G) \in \mathcal{G}$ is a doublecentered star, then $p(N, G)=\beta(N, G)$.

Proof: Let $(N, G) \in \mathcal{G}$ be a double-centered star with central positions $h$ and $i$. If $(N, G)$ is a star network, then the result follows from Lemma 4.1. Suppose $(N, G)$ is not a star network. By Lemma 4.2, $p_{j}(N, G)=\beta_{j}(N, G)$ for all $j \in I(N, G) \cup P(N, G)=N \backslash\{h, i\}$. Suppose without loss of generality that $\left|R_{(N, G)}(i)\right| \geq\left|R_{(N, G)}(h)\right|$. Define $\left(N^{\prime}, G^{\prime}\right)$ such that $N^{\prime} \supseteq N,\left|N^{\prime} \backslash N\right|=\left|R_{(N, G)}(i)\right|-\left|R_{(N, G)}(h)\right|$ and $G^{\prime}=G \cup\left\{\{h, j\} \mid j \in N^{\prime} \backslash N\right\}$. 
Lemma 4.2 implies that $p_{j}\left(N^{\prime}, G^{\prime}\right)=\beta_{j}\left(N^{\prime}, G^{\prime}\right)$ for all $j \in N^{\prime} \backslash\{h, i\}$. Anonymity implies that $p_{h}\left(N^{\prime}, G^{\prime}\right)=p_{i}\left(N^{\prime}, G^{\prime}\right)$, and thus with efficiency we have that $p_{i}\left(N^{\prime}, G^{\prime}\right)=$ $\frac{1}{2}\left(v^{G^{\prime}}\left(N^{\prime}\right)-\sum_{j \in N^{\prime} \backslash\{h, i\}} \beta_{j}\left(N^{\prime}, G^{\prime}\right)\right)=\beta_{i}\left(N^{\prime}, G^{\prime}\right)$.

Next, pending node addition implies that $p_{i}\left(N^{\prime}, G^{\prime}\right)-p_{i}(N, G)=p_{j}\left(N^{\prime}, G^{\prime}\right)-p_{j}(N, G)$ for all $j \in R_{(N, G)}(h) \backslash\{i\}$. (Note that such a $j$ exists because $(N, G)$ is not a star by assumption.) Since $p_{i}\left(N^{\prime}, G^{\prime}\right), p_{j}\left(N^{\prime}, G^{\prime}\right)$ and $p_{j}(N, G)$ are uniquely determined as the $\beta$ outcome, $p_{i}(N, G)$ is uniquely determined as the $\beta$ outcome $\beta_{i}(N, G)$. With efficiency then also $p_{h}(N, G)$ is uniquely determined as the $\beta$ outcome $\beta_{h}(N, G)$, and thus $p(N, G)=\beta(N, G)$.

The next step is to show uniqueness for acyclic networks. A sequence of positions $i_{1}, \ldots, i_{p}$ with $i_{1}=i_{p}, i_{k} \neq i_{\ell}$ for all $k, \ell \in\{1, \ldots, p-1\}$, and $\left\{i_{k}, i_{k+1}\right\} \in G$ for all $k \in\{1, \ldots, p-1\}$, is called a cycle in $(N, G)$. A network that contains no cycles is called an acyclic network. Note that a double-centered star is acyclic.

Lemma 4.4 If a power measure $p$ on $\mathcal{G}$ satisfies efficiency, anonymity, reasonability, non-neighbourhood independence and pending node addition, and $(N, G) \in \mathcal{G}$ is acyclic, then $p(N, G)=\beta(N, G)$.

Proof: Let $(N, G) \in \mathcal{G}$ be acyclic. If $(N, G)$ is a double-centered star network then the result follows from Lemma 4.3. Suppose $(N, G)$ is not a double-centered star network. By Lemma 4.2, $p_{i}(N, G)=\beta_{i}(N, G)$ for all $i \in I(N, G) \cup P(N, G)$.

Let $i, j \in N \backslash(I(N, G) \cup P(N, G))$ and define $G_{i}$ as in (5). Similarly, define the network $\left(G_{i}\right)_{j}=\left\{\{h, g\} \in G_{i} \mid h \in R_{\left(N, G_{i}\right)}(j)\right\}$ obtained as in (5) but for network $G_{i}$ and position $j$. Then $\left(G_{i}\right)_{j}$ is a double-centered star, and hence, $p_{j}\left(N,\left(G_{i}\right)_{j}\right)=\beta_{j}\left(N,\left(G_{i}\right)_{j}\right)$ by Lemma 4.3. Non-neighbourhood independence then implies that $p_{j}\left(N, G_{i}\right)=p_{j}\left(N,\left(G_{i}\right)_{j}\right)=\beta_{j}\left(N,\left(G_{i}\right)_{j}\right)=\beta_{j}\left(N, G_{i}\right)$.

Since for all $h \in I(N, G) \cup P(N, G)$ we have $h \in I\left(N, G_{i}\right) \cup P\left(N, G_{i}\right)$, it follows from Lemma 4.2 that for all such $h, p_{h}\left(N, G_{i}\right)=\beta_{h}\left(N, G_{i}\right)$. With efficiency we then conclude that $p_{i}\left(N, G_{i}\right)=v(N)-\sum_{j \in N \backslash\{i\}} \beta_{j}\left(N, G_{i}\right)=\beta_{i}\left(N, G_{i}\right)$.

Finally, non-neighbourhood independence implies that $p_{i}(N, G)=p_{i}\left(N, G_{i}\right)=$ $\beta_{i}\left(N, G_{i}\right)=\beta_{i}(N, G)$ and hence, $p(N, G)=\beta(N, G)$.

Finally, by adding edge cutting independence we can state our characterization of the $\beta$-measure. 
Theorem 4.5 A power measure $p$ on $\mathcal{G}$ is equal to the $\beta$-measure if and only if it satisfies efficiency, anonymity, reasonability, non-neighbourhood independence, edge cutting independence and pending node addition.

Proof: It is readily verified that the $\beta$-measure satisfies the six properties. Now, suppose that the power measure $p$ on $\mathcal{G}$ satisfies the properties, and let $(N, G) \in \mathcal{G}$. Let $i \in N$ and again define $G_{i}$ as in (5).

If $G_{i}$ is acyclic then $p_{i}\left(N, G_{i}\right)=\beta_{i}\left(N, G_{i}\right)$ by Lemma 4.4, and from nonneighbourhood independence we conclude that $p_{i}(N, G)=\beta_{i}(N, G)$. Otherwise, $\left(N, G_{i}\right)$ has at least one cycle, and each cycle has at least one edge $\{h, j\}$ not containing $i$. Delete such an edge, add two positions $r, s \notin N$ and add the edges $\{h, r\}$ and $\{j, s\}$ (i.e., consider the network $(N \cup\{r, s\},(G \backslash\{\{h, j\}\}) \cup\{\{h, r\},\{j, s\}\})$. Repeat this procedure until all cycles have been cut.

Clearly, this procedure ends in a finite number of steps with the resulting network $\left(N^{\prime}, G^{\prime}\right)$ being acyclic. Then $p_{i}\left(N^{\prime}, G^{\prime}\right)=\beta_{i}\left(N^{\prime}, G^{\prime}\right)$ by Lemma 4.4. Edge cutting independence implies that $p_{i}\left(N, G_{i}\right)=p_{i}\left(N^{\prime}, G^{\prime}\right)=\beta_{i}\left(N^{\prime}, G^{\prime}\right)=\beta_{i}\left(N, G_{i}\right)$. With nonneighbourhood independence it then follows that $p_{i}(N, G)=p_{i}\left(N, G_{i}\right)=\beta_{i}\left(N, G_{i}\right)=$ $\beta_{i}(N, G)$ and hence, $p(N, G)=\beta(N, G)$.

The degree measure satisfies all properties stated in Theorem 4.5 except efficiency. Instead, it satisfies an alternative normalization, which distributes twice the number of edges in a network.

Degree efficiency: For every $(N, G) \in \mathcal{G}$ it holds that $\sum_{i \in N} p_{i}(N, G)=2|G|$.

Note that the specific normalization is not essential for proving uniqueness in the proofs of Theorem 4.5 and the preceding lemmas. So, replacing efficiency by degree efficiency also yields uniqueness. Since all other properties of Theorem 4.5 are also satisfied by the degree measure, this yields a characterization of the degree measure.

Theorem 4.6 A power measure $p$ on $\mathcal{G}$ is equal to the degree measure if and only if it satisfies degree efficiency, anonymity, reasonability, non-neighbourhood independence, edge cutting independence and pending node addition.

\section{Concluding remarks}

We showed that the degree measure is the limit of a recursive procedure which starts with the $\beta$-measure, and in each step gives as output a new weighted $\beta$-measure, taking 
as input weights the weighted $\beta$-measure obtained in the previous step. Although the degree measure usually is considered to be a local measure, we thus have shown that it can be seen as a global measure within the context of power dependence theory (see Emerson (1962)). The degree measure also is a stationary power distribution in the sense that it satisfies (4).

On the other hand, Hendrickx, Borm, van den Brink and Owen (2005) take an alternative iterative approach. Instead of using weighted $\beta$-measures, they consider weighted Shapley values of the corresponding network power game. For various types of networks, they compute and interpret a Proper Shapley value as introduced by Vorob'ev and Liapunov (1998), which assigns to every network power game a particular weighted Shapley value such that these values are equal to the chosen weights. This yields a power measure $\pi$ satisfying

$$
\pi_{i}(N, G)=\sum_{j \in R_{(N, G)}(i)} \frac{\pi_{i}(N, G)}{\sum_{h \in R_{(N, G)}(j)} \pi_{h}(N, G)} \text { for all } i \in N
$$

In defining the conservative network power game we followed the game theoretic tradition to assign to every coalition the minimal power they can guarantee themselves. By definition, the dual game $\left(N, v^{*}\right)$ of a TU-game $(N, v)$ is given by $v^{*}(S)=v(N)-v(N \backslash S)$. It is easily verified that the dual game of the conservative network power game corresponding to $(N, G) \in \mathcal{G}$ assigns to every coalition of positions $S \subseteq N$ the total number of neighbours of $S$, and thus takes an optimistic approach to network power measurement. Since the Shapley value of a TU-game coincides with the Shapley value of its dual game, the $\beta$-measure also is equal to the Shapley value of this dual (optimistic) network power game $^{3}$. Moreover, linearity of the Shapley value implies that the $\beta$-measure equals the Shapley value of every convex combination of the conservative- and optimistic network power game.

Finally, we remark that the $\beta$-measure can be trivially extended to symmetric networks in which loops are allowed. On this class of graphs, the $\beta$-measure is characterized by the six properties of Theorem 4.5 and a property which states that the power of a position does not change if an adjacent loop is cut and replaced with a link to a copy of the original network.

\footnotetext{
${ }^{3}$ Since $v_{G}^{*}(\{i\})=\left|R_{(N, G)}(i)\right|=d_{i}(N, G)$, the optimistic network power game generalizes the degree measure. So, the Shapley value of the game that assigns to every coalition of positions its degree is equal to the $\beta$-measure.
} 


\section{References}

Berger, M.A. (1993), An Introduction to Probability and Stochastic Processes, SpringerVerlag, New York.

Borm, P., R. van den Brink, and M. Slikker (2002), "An Iterative Procedure for Evaluating Digraph Competitions", Annals of Operations Research, 109, 61-75.

Brink, R. van den, and P. Borm (2002), "Digraph Competitions and Cooperative Games", Theory and Decision, 53, 327-342.

Brink, R. van den, and R.P. Gilles (2000), "Measuring Domination in Directed Networks", Social Networks, 22, 1141-157.

Emerson, R.M. (1962), "Power-Dependence Relations", American Sociological Review, $27,31-41$.

Harsanyi, J.C. (1959), "A Bargaining Model for Cooperative n-Person Games", In Contributions to the Theory of Games IV (eds. Tucker A.W., and R.D. Luce), Princeton UP, Princeton pp 325-355.

Hendrickx, R., P. Borm, R. van den Brink, and G. Owen (2005), "The VL value for network games", Center Discussion Paper 2005-65, Tilburg University.

Shapley, L.S. (1953), "A Value for n-Person Games," Annals of Mathematics Studies 28 (eds. H.W. Kuhn and A.W. Tucker), Princeton University Press, 307-317.

Vorob'ev, N., and A. Liapunov (1998), "The Proper Shapley Value", In Game Theory and Applications Vol 4 (L.Petrosjan and M. Mazalov eds), Nova Science, New York, pp. 155-159. 